\title{
«Unser Draht zu den Spielern ist sehr direkt»
}

\author{
In dieser Woche laufen in Basel die «Swiss Indoors», eines der weltweit bedeutendsten \\ Hallen-Tennisturniere. Mit dabei ist seit über drei Jahrzehnten der Sportmediziner \\ Felix Marti. Im Interview gewährt der Turnier-Chefarzt Einblick in seine Aufgaben \\ und gibt auch persönliche Erinnerungen preis.
}

Interview: Bruno Kesseli

Herr Marti, Sie leiten an den Swiss Indoors das medizinische Team. Wie sieht die Organisation dieses sportlichen Grossanlasses im medizinischen Bereich aus?

\section{«Wir behandeln jeden Tag Spieler, wobei das}

Spektrum durchaus breit ist. [...] Es kommt auch vor, dass ich im Hotel der Spieler auf Visite gehe.»

\footnotetext{
* Association of Tennis Professionals, die Vereinigung der männlichen professionellen Tennisspieler. Die ATP organisiert die HerrentennisTurnierserie «ATP World Tour», zu der auch die «Swiss Indoors» in Basel zählen.
}

Unser Team umfasst drei Ärzte: Dr. Lucas Kälin, Orthopäde, Dr. Martin Tschan, Internist und Notfallmediziner, und mich selbst. Dazu kommen drei Physiotherapeuten, von denen einer von der ATP* gestellt wird. Letzterer ist eine Art Vertrauensperson der Spieler, da er fester Bestandteil des «Tenniszirkus» ist und die Spieler ihn schon von anderen Turnieren her kennen. Zum Team gehören auch ein Rettungssani- täter mit seinem ganzen Equipment sowie 3-4 Samariter. Unsere «Zentrale» ist ein Raum in den «Katakomben», in dem wir durchgehend erreichbar und einsatzbereit sind.

\section{Wie sieht der Einsatzplan für die drei Ärzte aus?}

Ein Arzt ist durchgehend anwesend, also ab Hallenöffnung bis ungefähr eine Stunde nach dem letzten Spiel. Während der Hauptspielzeiten, also wenn das Publikum in der Halle ist, sind stets alle drei Ärzte vor Ort.

Ihr Team ist nicht nur für die Spieler zuständig, sondern auch für die Behandlung von Zuschauern mit akuten medizinischen Problemen. Wie ist die Aufgabenverteilung in diesem Bereich?

Wenn jemand aus dem Publikum Probleme hat, schaut sich in der Regel ein Arzt mit dem Rettungs-

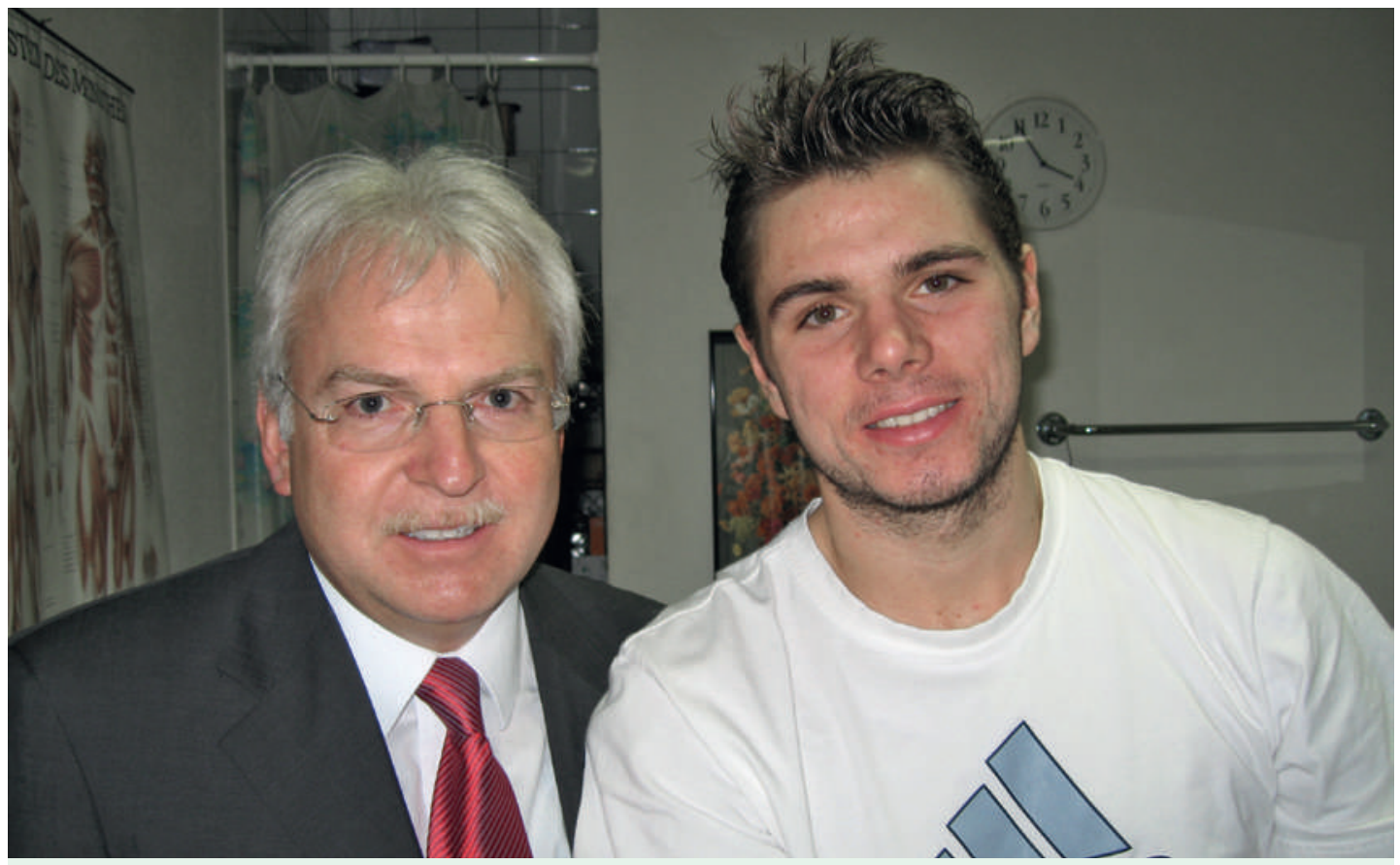

Unkomplizierter Kontakt zu den Spielern: Felix Marti (links) mit Stanislas Wawrinka, dem in diesem Jahr der Sprung unter die besten acht Tennisspieler der Welt gelang. 
sanitäter und einem Samariter die Situation vor Ort an und leitet die entsprechenden Massnahmen ein. Bei den Spielern ist das anders: Die kommen entweder vor oder nach dem Spiel zu uns, wenn sie ein Problem haben. Oder sie lassen uns in den Behandlungsraum rufen, wenn sie beispielsweise «getaped» werden, und schildern uns dort ihr Anliegen. Unser Draht zu den Spielern ist sehr direkt.

\section{Welches sind Ihre spezifischen Aufgaben als Chefarzt?}

Mir obliegen die gesamte Planung des medizinischen Bereichs in der Vorbereitungsphase und die Einsatzkoordination am Turnier selbst. Dazu bin ich erster Ansprechpartner für die Spieler, für die Turnierleitung, für das Publikum und auch für die ATP.

\section{«Sowohl Spieler als auch Coachs sind in Bezug auf Doping sehr sensibilisiert.»}

Welche Verpflichtungen haben Sie gegenüber der Spielergewerkschaft ATP?

Die ATP möchte im Vorfeld genau über die medizinische Organisation ins Bild gesetzt werden und erwartet nach Turnierende einen Bericht. Jede Spielerkonsultation wird von uns dokumentiert, was für die ATP sehr wichtig ist. Denn derselbe Spieler steht über das ganze Jahr an vielen verschiedenen Orten im Einsatz, an denen die medizinische Betreuung jeweils durch lokale Teams erfolgt. Nur eine saubere Dokumentation bietet Gewähr für eine kontinuierliche und kohärente Betreuung. Die Spieler sind natürlich über alle Behandlungen und Massnahmen umfassend informiert und bestätigen dies auch durch ihre Unterschrift.

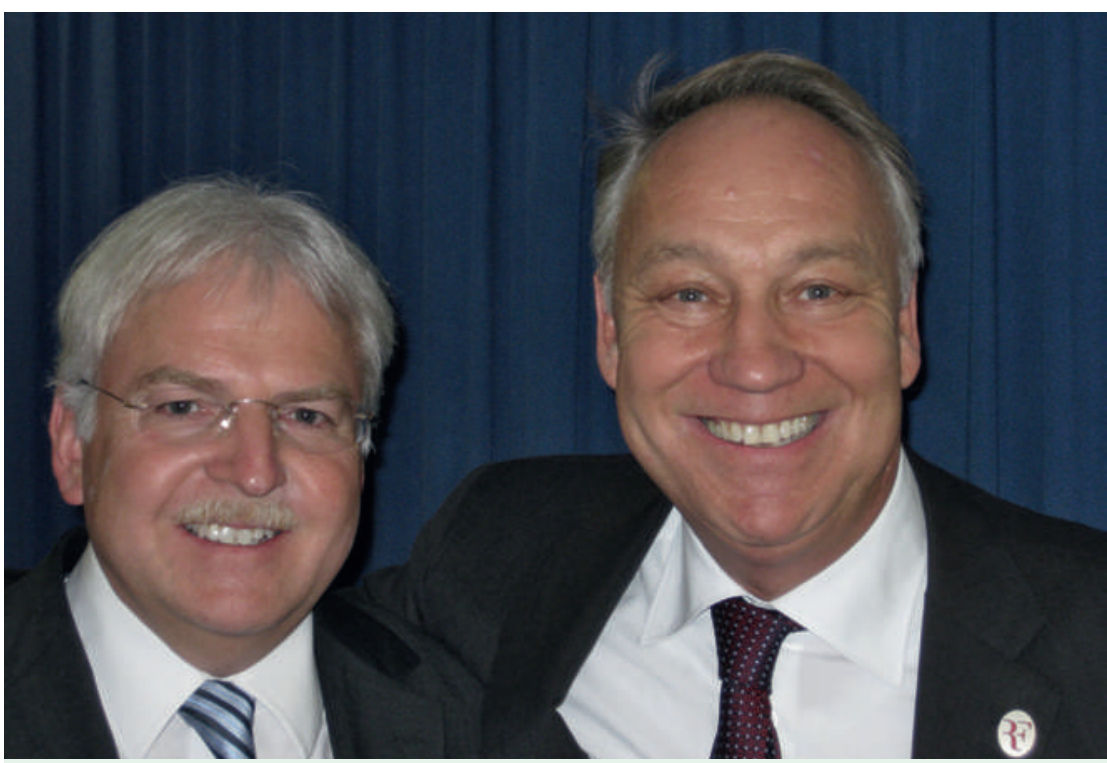

Perfekt organisiertes Turnier: Felix Marti mit Turnierpräsident Roger Brennwald (rechts).

\section{Zur Person}

Dr. med. Felix Marti ist Facharzt für Allgemeinmedizin und führt seit 26 Jahren eine eigene Praxis in Muttenz BL. Daneben ist er auf Sportmedizin spezialisiert und betreut in dieser Eigenschaft als Clubarzt die Spitzenfussballer des FC Basel und als Turnierarzt der «Swiss Indoors» professionelle Tennisspieler, die zu den Besten ihres Fachs zählen. Seit 1982 am Basler Tennisturnier im Einsatz, ist er mittlerweile der weltweit dienstälteste ATP-Turnierarzt. Felix Marti ist verheiratet und hat drei Kinder.

Sind Behandlungen von Spielern im Turnieralltag an der Tagesordnung oder eher die Ausnahme?

Wir behandeln jeden Tag Spieler, wobei das Spektrum durchaus breit ist. Sie müssen sich vor Augen halten, dass diese Spieler aus der ganzen Welt anreisen und einiges an Beschwerden mitbringen können, sei dies nun ein relativ harmloser grippaler Infekt, eine Gastroenteritis oder ein abklärungsbedürftiger Fieberzustand. Es kommt auch vor, dass ich im Hotel der Spieler auf Visite gehe, aber während des Turniers erfolgen die Konsultationen in der Regel in unserem Sanitätszimmer in der St. Jakobshalle.

Haben Spitzenspieler für sportspezifische Probleme auch ihre eigenen Ärzte dabei?

Nein, eigene Ärzte bringen sie nicht mit, höchstens einen Physiotherapeuten. Gelegentlich konsultieren uns auch ihre Coachs. In der Regel kommen die Spieler aber einfach direkt bei uns vorbei - das ist bei uns sehr unkompliziert.

Nehmen wir an, Rafael Nadal kommt zu Ihnen mit Kniebeschwerden - angesichts seiner komplexen Vorgeschichte und seines Status sicher keine einfache Situation. Wie sähe in einem solchen Fall das Vorgehen aus?

Es stellt sich zunächst die Frage, ob er mit der Verletzung spielen kann oder nicht. Das Knie wird im Hinblick darauf von uns untersucht und beurteilt. Wenn wir zu keinem abschliessenden Urteil kommen, werden weitere Abklärungen veranlasst, zum Beispiel ein MRI. Danach kann es allenfalls zu einem Rückzug aus dem Turnier kommen, was in den entsprechenden Formularen der ATP genau dokumentiert werden muss. Solche Untersuchungen und Entscheide finden aber nicht auf dem Platz statt, sondern vor oder nach dem Spiel.

Wie ist der Ablauf, wenn während des Spiels Probleme auftreten?

Der Spieler verlangt ein «medical timeout». In diesem Fall begibt sich zunächst der Physiotherapeut der ATP auf den Platz und beurteilt die Situation. Stellt sich die Frage eines Spielabbruchs, wird zusätzlich ein Arzt beigezogen. 
Sie überblicken als Turnierarzt einen Zeitraum von über drei Jahrzehnten. Gab es Vorkommnisse auf und neben dem Platz, die Ihnen speziell in Erinnerung geblieben sind?

Medizinisch denkwürdig war für mich beispielsweise eine akute Hämorrhoidalthrombose, die während des Spiels zu einer sichtbaren Blutung aufgrund eines geplatzten Hämorrhoidalknotens führte. Daneben gab es auch eher skurrile Erlebnisse: So klagte ein Spieler über starke Schmerzen in der Leistengegend und wollte wegen einer vermeintlichen Leistenzerrung aufgeben. Als ich ihn unter der Tribüne untersuchte, stellte ich fest, dass er extrem enge Unterhosen trug,

\section{«Ich bin klar der Meinung, dass Spieler wie Connors,} Noah, Edberg oder Becker auch schon absolut guttrainierte Athleten auf Top-Niveau waren.»

und riet ihm, diese zu wechseln. Danach spielte er sein Match beschwerdefrei zu Ende. Gut erinnere ich mich auch an die Besuche von Stefan Edberg in meiner Praxis. Nachdem er sich vor Turnierbeginn an einem Rechaud eine Verbrennung mit Brandblasen am Daumen der Schlaghand zugezogen hatte, kam er zweimal täglich vorbei, um sich für das Training beziehungsweise das Spiel vorbereiten zu lassen. Das ging gut - er gewann das Turnier.

\section{Konsultationen in Ihrer Praxis dürften aber eher die Aus-} nahme sein?

Es kommt immer wieder vor, dass sich Spieler kurz vor einem Turnier aus medizinischen Gründen abmelden. Bis vor kurzem waren sie in einem solchen Fall dazu verpflichtet, die Spielunfähigkeit vom Turnierarzt bestätigen zu lassen. Aus diesem Grund besuchten mich beispielsweise schon Pete Sampras, Boris Becker und Rafael Nadal vor dem Turnier in

\section{«Swiss Indoors Basel»}

Die Geschichte der «Swiss Indoors Basel» begann 1970 in einer Traglufthalle und mit einer Armbanduhr als Preis für den Sieger. Heute zählt das in der Kategorie Formel 500 eingestufte Turnier der ATP World Tour mit einer Preisgeldsumme von knapp 2 Millionen Euro zu den bedeutendsten Hallen-Tennisanlässen der Welt. Eine hohe Dichte an Spitzenspielern gilt seit langem als Markenzeichen der «Swiss Indoors Basel». Selbst gemessen an diesem Massstab ist das Feld in diesem Jahr mit der aktuellen Weltnummer 1, Rafael Nadal, sowie weiteren fünf Spielern aus den «Top Ten» - darunter die beiden Schweizer Roger Federer und Stanislas
Basel. Nadal musste dazu eigens aus Mallorca anreisen, Becker aus München. Sampras hatte immerhin das Glück, zu diesem Zeitpunkt schon in Europa $\mathrm{zu}$ sein und nicht den Atlantik überqueren zu müssen. Heute ist dieser Reiseaufwand dank besserer Vernetzung und Dokumentation nicht mehr nötig.

\section{Ist Doping für Sie als Turnierarzt ein Thema?}

Im Rahmen des Turniers finden regelmässig Dopingkontrollen statt. Wir müssen dafür nur die Räumlichkeiten zur Verfügung stellen, die Durchführung obliegt der WADA**. In den letzten fünf Jahren waren diese Kontrollen sehr häufig. Es sind natürlich viele Gerüchte über Doping im Tennissport im Umlauf. Was den tatsächlichen Gebrauch verbotener leistungsfördernder Substanzen betrifft, ist die Faktenlage aber sehr dünn. Sowohl Spieler als auch Coachs sind jedoch in Bezug auf Doping sehr sensibilisiert. Wenn ich einem Spieler ein Medikament gebe, werde ich mit Sicherheit gefragt, ob es nicht auf der Dopingliste stehe, was wir natürlich jeweils bestätigen können.

Wie haben sich das Basler Turnier und der Tennissport in den vergangenen Jahrzehnten in Ihrer Wahrnehmung entwickelt?

«Es ist sicher so, dass die Perfektion, mit der Federer Tennis spielt, die Verletzungsanfälligkeit minimiert.»

In Bezug auf die Turnierorganisation ist die Professionalisierung und Perfektionierung aller Abläufe eindrücklich. Die Swiss Indoors sind eines der grössten Hallenturniere der Welt, und alles ist bis ins kleinste Detail durchorganisiert und klappt reibungslos. Was das Tennis angeht: Der Sport ist in dieser Zeit noch schneller und athletischer geworden. Ich bin aber klar der Meinung, dass Spieler wie Connors, Noah, Edberg oder Becker auch schon absolut guttrainierte Athleten auf Top-Niveau waren. Diesbezüglich sind für mich die Unterschiede zu den heutigen Spitzenspielern nicht so gross, wie sie häufig postuliert werden.

Welche Spieler, die Sie erlebt haben, haben Sie persönlich am meisten beeindruckt?

Der eindrücklichste Spieler - auch weil ich einen Grossteil seiner sportlichen Entwicklung miterleben durfte - ist für mich Roger Federer. Er begann ja bei uns als ganz normaler Balljunge, schreibt als «Jahrtausendtalent» für die Schweiz Tennisgeschichte und bleibt trotzdem ein ganz normaler Basler. 


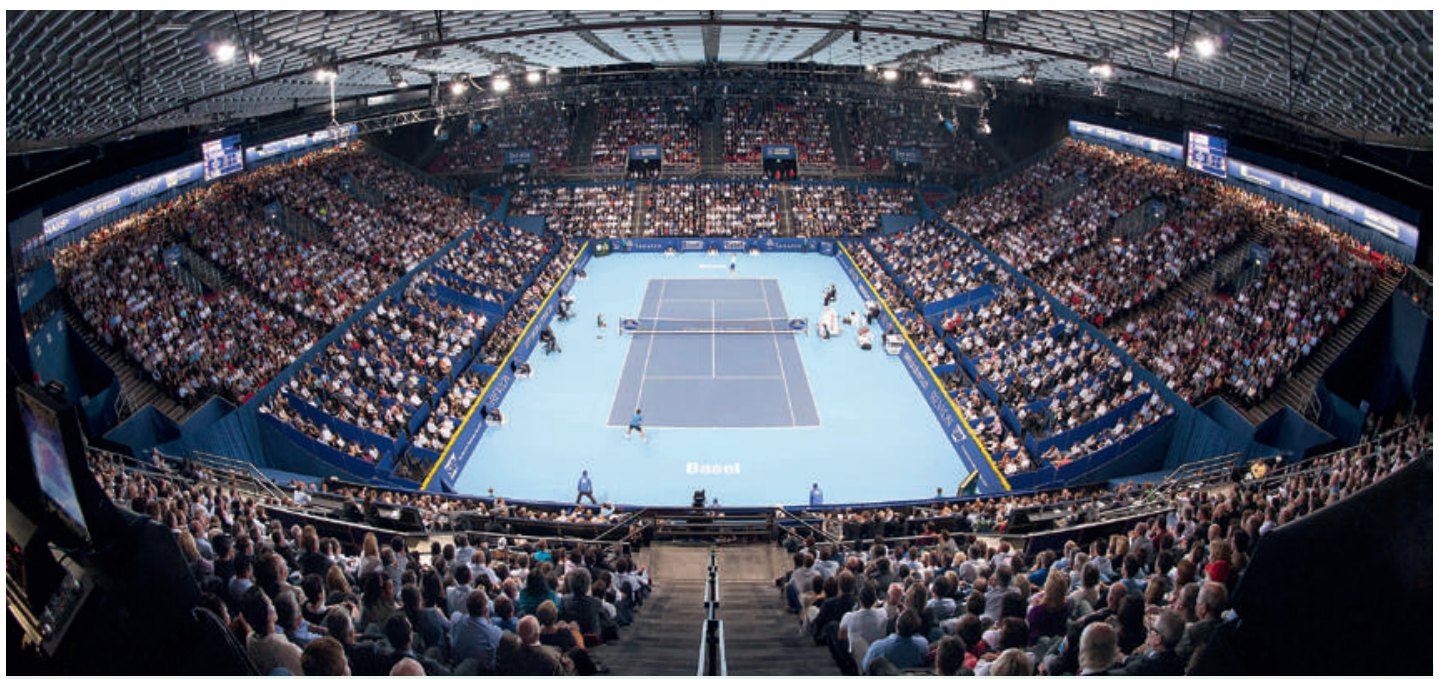

Eindrückliche Tennis-Arena: Rund 8500 Zuschauer können die Spiele in der St. Jakobshalle verfolgen. Foto: Peter Hauck

Bei Federer hört man oft die Einschätzung, dass er aufgrund seiner harmonischen Bewegungsabläufe viel weniger verletzungsanfällig ist als andere Spieler, die technisch weniger ausgereift sind. Sehen Sie dies auch so?

Es ist sicher so, dass die Perfektion, mit der Federer Tennis spielt, die Verletzungsanfälligkeit minimiert. Technisch weniger begabte Spieler, die mehr vom Kampf leben - wobei Federer fraglos auch ein Kämpfer ist - haben diesbezüglich wohl schlechtere Karten. Ich denke schon, dass ein Spieler vom Schlage eines Rafael Nadal seine Sehnenansätze mit seinem kampfbetonten Tennis stärker belastet als Federer.

Da Sie auch die Fussballer des FC Basel betreuen:
Können Sie einen kurzen Quervergleich der beiden Sportarten bezüglich Verletzungsrisiko ziehen?

Das Risiko von Verletzungen ist im Tennis geringer als im Fussball. Das hat einen einfachen Grund: Der Gegner befindet sich im Tennis auf der anderen Seite des Netzes. Im Fussball entstehen die meisten Verletzungen im direkten Kontakt mit dem Gegenspieler. Aus dieser Perspektive ist der Schiedsrichter der wichtigste Mann auf dem Platz: Er muss das Spiel so leiten, dass die Knochen der Spieler geschont werden. Im Tennis spielen heutzutage übrigens praktisch alle Spieler mit prophylaktischen Tapes im Bereich der Sprunggelenke, eine sehr sinnvolle Massnahme.

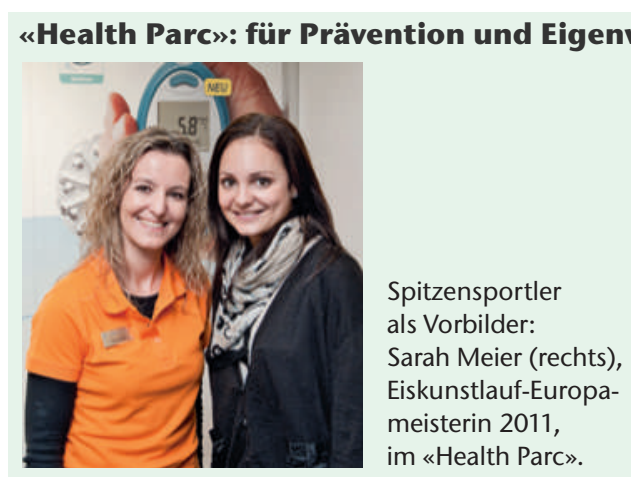

Die Besucher der Swiss Indoors bekommen nicht nur Spitzentennis von durchtrainierten Athleten zu sehen, sie erhalten auch die Gelegenheit, Anhaltspunkte zu ihrer eigenen körperlichen Verfassung zu gewinnen. Bereits seit einigen Jahren ist ein kostenloser «Health Check» für alle Besucher Bestandteil des Rahmenkonzepts der Swiss Indoors. Die Idee des von verschiedenen Partnern aus dem Gesundheitsbereich mitgetragenen «Health Parc» ist

\section{ortung sensibilisieren}

es, das Publikum für Fragen im Zusammenhang mit Gesundheit und Eigenverantwortung zu sensibilisieren und damit einen Beitrag zur Prävention zu leisten. Zum Angebot gehören ein Sehund ein Hörtest, eine Hautanalyse mit Beurteilung von Nävi («Muttermalen»), ein «Risikotest Lunge», ein Sprungkrafttest sowie ein «Bodycompositionstest» (Messung der individuellen Verteilung von Fett- und Muskelmasse).

Die Teilnehmerzahlen der letzten drei Jahre zeigen, dass dieses Angebot bei den Zuschauern gut ankommt: Durchschnittlich 80-100 Besucher liessen sich pro Tag testen, wobei Frauen etwas stärker vertreten waren als Männer (55\%:45\%). Der Test spricht offenbar alle Altersgruppen an: Rund 20\% der Teilnehmer waren jünger als 30 Jahre, 10\% älter als 60 Jahre. Am stärksten vertreten war mit rund $40 \%$ die Gruppe der 45-60-Jährigen. Es ist zu vermuten, dass diese Altersverteilung etwa dem Besucherprofil der Swiss Indoors entspricht. 